This item was submitted to Loughborough's Research Repository by the author.

Items in Figshare are protected by copyright, with all rights reserved, unless otherwise indicated.

\title{
Characterizing the switching transitions of an adsorbed peptide by mapping the potential energy surface
}

PLEASE CITE THE PUBLISHED VERSION

https://doi.org/10.1021/acs.jpcb.7b10319

\section{PUBLISHER}

(C) American Chemical Society

\section{VERSION}

AM (Accepted Manuscript)

\section{PUBLISHER STATEMENT}

This work is made available according to the conditions of the Creative Commons Attribution-NonCommercialNoDerivatives 4.0 International (CC BY-NC-ND 4.0) licence. Full details of this licence are available at: https://creativecommons.org/licenses/by-nc-nd/4.0/

\section{LICENCE}

CC BY-NC-ND 4.0

\section{REPOSITORY RECORD}

Ross-Naylor, James A., M. Mijajlovic, Hu Zhang, and Mark J. Biggs. 2019. "Characterizing the Switching Transitions of an Adsorbed Peptide by Mapping the Potential Energy Surface". figshare. https://hdl.handle.net/2134/27899. 


\section{Characterizing the Switching Transitions of an}

\section{Adsorbed Peptide by Mapping the Potential Energy \\ Surface}

James A. Ross-Naylor, Milan Mijajlovic $†, H u$ Zhang and Mark J. Biggs* $\neq$

School of Chemical Engineering, The University of Adelaide, Adelaide, South Australia, 5005

Australia

*Corresponding author. Phone: +44 (0)1509 222 600. E-mail: m.biggs@lboro.ac.uk.

†Present address: School of Chemical Engineering and Advanced Materials, Newcastle University, NE1 7RU, United Kingdom

\$Present address: School of Science, Loughborough University, Leicestershire, LE11 3TU, United Kingdom

The manuscript was written through contributions of all authors. All authors have given approval to the final version of the manuscript. 


\section{ABSTRACT}

Peptide adsorption occurs across technology, medicine and nature. The functions of adsorbed peptides are related to their conformation. In the past, molecular simulation methods such as molecular dynamics have been used to determine key conformations of adsorbed peptides. However, the transitions between these conformations often occur too slowly to be modeled reliably by such methods. This means such transitions are less well understood. In the study reported here, discrete path sampling is used for the first time to study the potential energy surface of an adsorbed peptide (polyalanine) and the transition pathways between various stable adsorbed conformations that have been identified in prior work by two of the authors (Mijajlovic, M.; Biggs, M. J. J. Phys. Chem. C 2007, 111, 15839-15847). Mechanisms for the switching of adsorbed polyalanine between the stable conformations are elucidated along with the energetics of these switches.

\section{INTRODUCTION}

The interaction of peptides and proteins with solid surfaces has attracted significant attention over many years. ${ }^{1-3}$ Central to much of this prior work has been the application of peptide and protein adsorption in nanotechnology. ${ }^{4-6}$ For example, nanoparticles have been implicated in the denaturing of proteins in the human body, leading to toxicity. ${ }^{7}$ More positively, peptides may be used to form nanoparticle assemblies, ${ }^{8}$ or to functionalize nanomaterials for applications such as cancer diagnosis and treatment, ${ }^{9-11}$ biosensing ${ }^{12-14}$ and drug delivery. ${ }^{15}$ Peptide adsorption is also of relevance in biology and medicine. For example, it is implicated in the fouling and degradation of medical implants, ${ }^{16}$ and may play a role in the fibrillation of proteins associated with degenerative brain diseases such as Alzhemier's. ${ }^{17-18}$ 
Given the widespread applications of peptide and protein adsorption, it is desirable to understand the molecular processes involved in this phenomenon. All-atom molecular dynamics has been widely used to study preferred structures of adsorbed peptides, ${ }^{19-20}$ and studies in recent years have succeeded in estimating the free energy of adsorption, ${ }^{21-23}$ and proposing mechanisms for the peptide adsorption process. ${ }^{24-26}$ However, all-atom molecular dynamics is only useful for adsorption events lasting up to the order of a microsecond, and as such, slower but no less important processes, such as the change in secondary structure of an already adsorbed peptide, are as yet less well understood. ${ }^{3,20} \mathrm{Up}$ until the present time, these processes have only been investigated using spectroscopic experimental methods and coarse-grained simulations, which offer less definitive understanding than all-atom models..$^{3,20}$

Discrete path sampling ${ }^{27}$ is a promising all-atom methodology for investigating transitions between two conformations of a system that are too slow to be probed by all-atom molecular dynamics. In short, this approach involves finding stationary points on the potential energy surface (PES) - that is, the minima (stable configurations) and saddle points (maximum-energy transition states) - between two conformations of interest. Transition pathways are then approximated as sequences of stationary points between the desired endpoints, and rate constants may be estimated using occupational probabilities derived from harmonic densities of states. ${ }^{27-29}$ This methodology has been applied to study the rearrangements of clusters ${ }^{30-31}$ and biomolecules $^{32-34}$ in studies dating up to the recent past. However, to the best of our knowledge, it has not been used to investigate changes in conformation of adsorbed peptides.

In a previous study ${ }^{35}$ in which molecular modeling was used to study the adsorption of polyalanine at gas-solid interfaces, some of the authors here showed that the polyalanine can take on one of the three helical structures illustrated in Figure 1 depending on the strength of the 
solid-peptide interaction, and that switching occurs between these structures at specific solidpeptide interaction strengths. In the study reported here, discrete path sampling was used to elucidate the transitions between these conformations within the vicinity of the switching points. The nature of the PES of the system was also explored as a function of the solid-peptide interaction strength. In this report, we first outline the molecular model and methods used along with the details of the study undertaken. The switching energies, transition paths and associated rate constants are then presented and discussed. The report ends with a summary of key conclusions and an outline of future work.

(a)

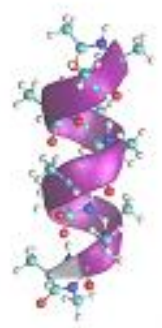

(b)
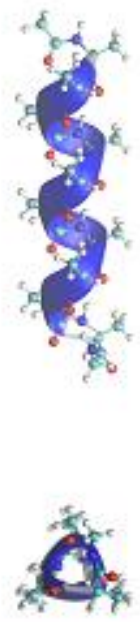

(c)
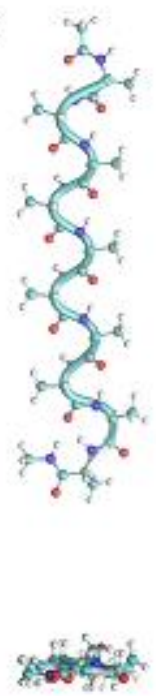

Figure 1. (a) Top and side views of the three helical forms taken by 10-alanine depending upon the strength of the interaction between the peptide and the solid surface: ${ }^{35}$ (a) $\alpha$-helix, which is found when the solid interacts weakly with the peptide (and also when in the gas phase); (b) $310^{-}$ helix, which is found when the solid interacts more strongly with the peptide; and (c) 27-helix, which is found when the interaction between the solid and peptide is high. The structures were generated using Visual Molecular Dynamics. ${ }^{36}$ 


\section{METHODOLOGY}

\subsection{MODEL}

The model is essentially composed of a molecular model of polyalanine above a solid surface. The details of this model were set to replicate those of Mijajlovic and Biggs. ${ }^{35}$ The polyalanine molecules were capped by an acetyl group $\left(\mathrm{CH}_{3} \mathrm{CO}\right)$ at the $\mathrm{N}$-terminus and an amino-methyl group $\left(\mathrm{NHCH}_{3}\right)$ at the $\mathrm{C}$-terminus. Intramolecular interactions were modeled using the Amber94 force field..$^{37}$

The interaction between the peptide and solid surface was modeled by the Steele potential: ${ }^{38}$

$$
E_{A u}=2 \pi \rho \sum_{j} \sum_{l=0}^{L-1} \varepsilon_{s j} \sigma_{s j}{ }^{2}\left[\frac{2}{5}\left(\frac{\sigma_{s j}}{z_{j}+l \Delta}\right)^{10}-\left(\frac{\sigma_{s j}}{z_{j}+l \Delta}\right)^{4}\right]
$$

where $l=0, \cdots, L-1$ is a counter over the layers of solid atoms in the surface up to the maximum, $L, \rho$ the density of atoms in each of the layers, $\Delta$ the distance between the layers, $z_{j}$ the perpendicular distance of peptide atom- $j$ from the surface, and $\varepsilon_{s j}$ and $\sigma_{s j}$ the associated Lennard-Jones energy and length parameters, respectively. The solid surface parameters for Au [111], which are summarized in Table 1 , were derived from the literature. ${ }^{39-40}$ Specifically, the Lorentz-Berthelot rules were used to calculate the surface Lennard-Jones parameters, $\varepsilon_{s}$ and $\sigma_{s}$, using the literature parameters for $\mathrm{CH}_{2}$ interacting with $\mathrm{Au},{ }^{39}$ and these same rules were then applied with the Amber94 force field parameters to calculate $\varepsilon_{s j}$ and $\sigma_{s j}$. A range of surfacepeptide interaction strengths, $E_{s}$, were investigated through a simple expedient of multiplying the potential energy arising from the Steele model by a factor, $E_{S} / E_{A u}$, which we call the surface energy ratio.

Table 1. Steele model parameters for $\mathrm{Au}$ [111].

\begin{tabular}{|l|l|}
\hline parameter & value \\
\hline
\end{tabular}




\begin{tabular}{|l|l|}
\hline$\rho$ & 0.13886 atoms $/ \AA^{2}$ \\
\hline$\varepsilon_{S}$ & $0.0905 \mathrm{kcal} / \mathrm{mol}$ \\
\hline$\sigma_{S}$ & $3.359 \AA$ \\
\hline$\Delta$ & $2.3545 \AA$ \\
\hline$L$ & 2 \\
\hline
\end{tabular}

\subsection{METHODS}

The study is split into two parts. The first, which essentially replicates the previous work reported in ref. 35 using a different method to identify the minimum energy polyalanine conformations, involved determining which of the three helix forms is the most favored for a range of surface energies. This then underpinned identification of the surface energies where switching between two helix forms occurs.

The minimum energy polyalanine conformations and associated energy were identified for each of the helix forms as follows. Using a canonical form of the helix in question - either $\alpha$-, 310- or 27 -helix - the limited-memory Broyden-Fletcher-Goldfarb-Shanno (LBFGS) algorithm ${ }^{41}$ was used to find the minimum energy conformation starting from 1296 initial conformations that were generated by systematically varying the two angles defined in Figure 2 by increments of $10^{\circ}$ between the possible limits (where this process resulted in a peptide atom being within $1 \AA$ of the surface, the peptide was shifted normal to the surface until this minimum distance was satisfied). The canonical forms of the $\alpha-, 33_{10^{-}}$and 27 -helixes were generated by setting all the backbone dihedral angles to $\left(\psi=-60^{\circ}, \phi=-45^{\circ}\right),\left(-55^{\circ},-15^{\circ}\right)$ and $\left(-75^{\circ}, 70^{\circ}\right)$, respectively.

The most favored helix form for a given surface energy is that identified as having the lowest potential energy for the surface energy. The switching point between two helix forms is that surface energy where the energies of two different forms are identical. 


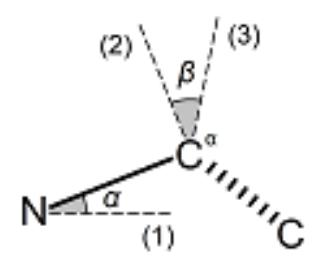

Surface

Figure 2. The angles, $\alpha$ and $\beta$, that were varied to generate initial adsorbed polyalanine configurations. The atoms shown belong to the residue nearest to the $\mathrm{N}$-terminus. The viewing plane contains the $\mathrm{N}-\mathrm{C}^{\alpha}$ bond and is perpendicular to the surface. The labeled lines are (1) parallel to the surface in the viewing plane, (2) perpendicular to the $\mathrm{N}-\mathrm{C}^{\alpha}$ bond in the viewing plane, and (3) normal to both bonds.

The second part of the study reported here was concerned with identifying the switching pathway and associated energy barrier for the two switches (i.e. $\alpha \rightarrow 3_{10}$ and $3_{10} \rightarrow 2_{7}$ ). As illustrated in the flow diagram shown in Figure S1 in the Supporting Information, this involved the application of discrete path sampling ${ }^{27}$ to extensively enumerate the stationary points (i.e. minima and saddle points) on the PES of the systems in the vicinity of the switching points and the pathways between them. The details of this process are outlined in the remainder of this section below.

The iterative enumeration of stationary points and pathways between them for a PES started by repeatedly using basin-hopping ${ }^{42}$ combined with simulated annealing (SA-BH) to expand the database of minima identified through the first part of this study. SA-BH involves randomly perturbing all backbone dihedral angles up to a maximum followed by relaxing the resulting structure using the LBFGS algorithm. ${ }^{41}$ The Metropolis criterion is then applied to determine whether to accept or reject the step, and this procedure is repeated for a specified number of 
steps. The SA component of the algorithm sees the temperature used in application of the Metropolis criterion gradually reduce over the course of the simulation from an initial high value. The maximum step change in the dihedral angles was initially set at $360^{\circ}$ and then adjusted regularly to maintain an acceptance ratio of 0.5 .

Following considerable expansion of the minima database using SA-BH, a variety of selection schemes were used to choose pairs of minima to be used as starting points for a saddle point search. Details of the saddle point searches are provided in the following paragraph. Firstly, the DIJINIT ${ }^{43}$ scheme was used to iteratively construct a putative pathway of connected stationary points between the minima associated with the two helix forms that define the switch being considered. Once this putative pathway was established, further selection schemes were applied iteratively to find additional stationary points that may be used to construct more energetically accessible pathways between the endpoint minima. These schemes included SHORTCUT, ${ }^{44}$ DIJPAIR ${ }^{45}$ and UNTRAP, ${ }^{45}$ which all attempt to shorten the putative minimum energy pathway and decrease the energy barrier, by finding unknown stationary points near the pathway. The NEWCONNECTIONS ${ }^{46}$ scheme was also employed, which iteratively selects each known minimum below the energy barrier of the current minimum energy pathway and applies a small random perturbation. An additional scheme, which we call CONNUSERMIN, was also used in this study to connect a user-specified list of minima with their closest neighbors in Euclidean space; this was used to cross-check the putative pathways between the same helix forms at different surface energies, ensuring structures along the transition path at one surface energy ratio were adequately sampled at the others.

The identification of saddle points between pairs of minima first involved use of the doubly nudged elastic band (DNEB) method $^{47}$ to identify saddle point candidates between the two 
chosen minima. Each candidate was then in turn refined using eigenvector following. ${ }^{48}$ Following the precise location of a saddle point, the LBFGS algorithm was once again used to identify the two minima adjacent to the saddle point; if any of these minima were previously unknown, they were added to the database of stationary points. When using the NEWCONNECTIONS scheme, the DNEB method was skipped entirely and eigenvector following was applied directly to the perturbed minimum.

After applying any given scheme, the KSHORTESTPATHS algorithm ${ }^{49}$ was used to determine the lowest energy pathway and calculate the rate constant. ${ }^{29}$ The refining of the putative switching pathway continued until the change in the rate constant of the minimum energy pathway between the two forms of helix of interest did not substantially change with the number of discovered minima. As illustrated in Figure S2 in Supporting Information, the rate of change of the rate constant with the number of discovered minima approached zero for both switches, suggesting that the PES were well-mapped near the helixes of interest and the limiting rate constants can be associated with the switching pathway.

The various algorithms mentioned above were accessed using the software of Wales and coworkers, which is freely available on their website. ${ }^{46}$ A summary of these algorithms and schemes is given in the Supporting Information. Their usage is this study required interfacing the software with the CHARMM program, ${ }^{50}$ which was used to evaluate the potential energy for a given peptide conformation. The CHARMM program was modified to include the Steele potential.

\subsection{STUDY DETAILS}

As per the authors' previous study, ${ }^{35}$ polyalanine molecules of length ranging from 6 to 12 residues were considered for surface energies ranging from $E_{s} / E_{A u}=0.0$ (i.e. gas-phase 
polyalanine) to $E_{S} / E_{A u}=5.5$ at increments of 0.1 . The structures obtained from minimization were classified as $\alpha-, 3_{10}$ - or 27 -helixes or otherwise, under the criterion that at least two thirds of the residues must have both backbone dihedral angles within $\pm 20^{\circ}$ of the values associated with the canonical values given above.

The $\alpha \rightarrow 3_{10}$ and $3_{10} \rightarrow 2_{7}$ transitions were each studied for 10 -alanine at three surface energy ratios near to the switching point. The multiple energies were studied to investigate whether there were any significant changes to the transition pathways either side of the switching point.

Each SA-BH simulation was run for a total of 50,000 steps, with an initial temperature of $k_{B} T=5.0 \mathrm{kcal} / \mathrm{mol}$, which was decremented by $8 \times 10^{-3} \%$ after each step. The maximum step size was adjusted up or down by $5 \%$ every 50 steps if the acceptance ratio for the last 50 steps was greater than or less than 0.5 , respectively.

The DNEB method was applied with 10 images and a maximum of 300 iterations. The LBFGS algorithm, which was used in both the DNEB and eigenvector following methods, was implemented with a memory of the last 4 iterations, a maximum step size of 0.4 , initial guesses for the diagonals of the Hessian matrix of 0.1 , and a convergence criterion of the RMS gradient not exceeding $10^{-7}$.

Perturbations to minima for the NEWCONNECTIONS scheme were randomized to a maximum of $0.01 \AA$ for each Cartesian coordinate. Paths and rate constants calculated using the KSHORTESTPATHS algorithm were evaluated at a temperature of $k_{B} T=0.3 \mathrm{kcal} / \mathrm{mol}$.

\section{RESULTS AND DISCUSSION}

\subsection{SWITCHING BEHAVIOUR OF POLYALANINE MOLECULES}

Figure 3 shows for 10 -alanine the variation of the potential energy of the minimum-energy $\alpha$-, $3_{10}$ - and $27^{-}$helixes with the surface energy ratio; the variation is similar for the other polyalanine 
molecules investigated here. The helix structure with the lowest energy for a given surface energy ratio is the most favored at that surface energy. Thus, in line with our previous study, ${ }^{35}$ the $\alpha$-helix is the most favored in the absence of the surface (i.e. in the gas phase) and when the solid surface interacts weakly with the peptide, the $2{ }_{7}$-helix is preferred at the highest surface energies investigated here, and the 310 -helix is favored for energies between these two extremes. There are two switching points: where the $\alpha$ - and 310 -helixes are equally favored at a low surface energy ratio, and where the $3_{10-}$ and 27 -helixes are equally favored at a high surface energy ratio. For 10-alanine, the $\alpha \rightarrow 3_{10}$ switching point is $E_{S} / E_{A u}=0.121$, whilst that of the $3{ }_{10} \rightarrow 2_{7}$ switch is $E_{S} / E_{A u}=4.400$.

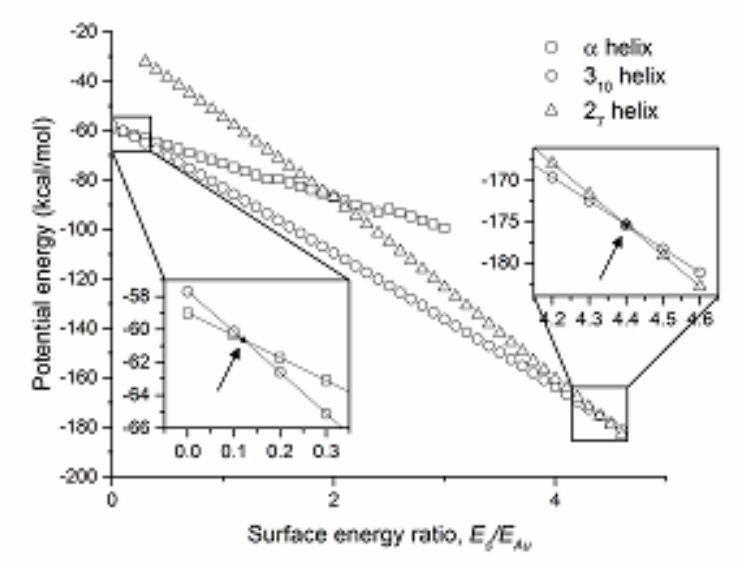

Figure 3. Variation of the potential energy of the $\alpha$-, $310^{-}$and 27 -helix structures of 10 -alanine with the surface energy ratio. Insets show the behavior near the $\alpha \rightarrow 3_{10}$ and $33_{10} \rightarrow 27$ switching points. Lines shown in the insets are used to locate the switching points, which are at the intersections of the lines indicated by the arrows.

Figure 4 shows the switching point surface energies as a function of the size of the polyalanine molecule. The variation seen in this figure is similar to that obtained by Mijajlovic and Biggs (Figure 9(a) in their work). ${ }^{35}$ However, there are two clear differences. The first is the preference 
for the $3{ }_{10}$-helix in the gas phase and lower surface energies when the number of residues drops below 10. This preference in the gas phase for short polyalanines is not inconsistent with earlier results of others. ${ }^{51-52}$ It is also in line with the work of Park and Goddard, ${ }^{53}$ who showed that polyalanine $\alpha$-helixes are stabilized by dipole-dipole interaction energy that increases with the number of residues. This result is corroborated by other studies that show $\alpha$-helical global minima for long polyalanines. ${ }^{54-57}$ More recently, studies of polyalanine and polyalanine derivatives adsorbing on solid surfaces have indicated the formation of $\alpha$-helixes through simulation (via a course-grained Monte Carlo study), ${ }^{58}$ and both $\alpha$-helixes ${ }^{59-60}$ and $33_{10}$-helixes ${ }^{60}$ through experiment (via synthesis of self-assembled monolayers), although it must be noted that none of these studies precisely replicate the system studied here. The second difference between the results obtained here and those of Mijajlovic and $\operatorname{Biggs}^{35}$ is the absolute values of the switching energies: they are significantly higher and lower for $3_{10} \rightarrow 27$ and $\alpha \rightarrow 3_{10}$ switches, respectively.

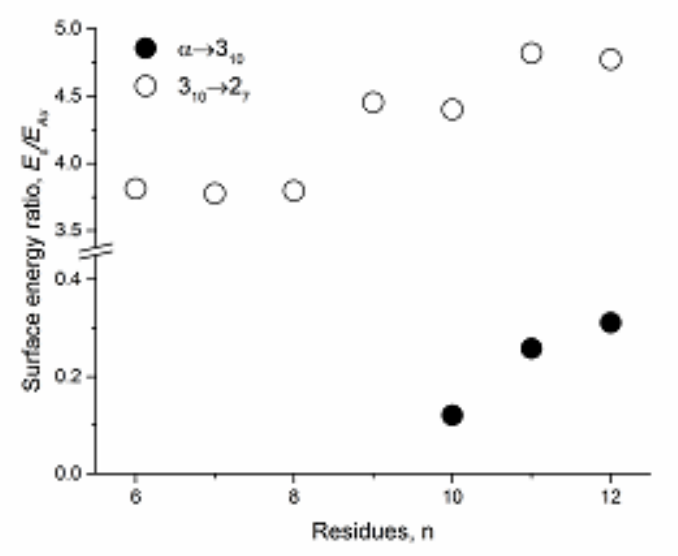

Figure 4. Variation of the switching surface energy ratio with the polyalanine length for the $\alpha \rightarrow 3_{10}$ and $3_{10} \rightarrow 27$ switches. 
The differences observed between the results obtained in the study reported here and the study of Mijajlovic and Biggs ${ }^{35}$ are easily explained by one fundamental difference in the methodologies used. While the force field and surface models are identical, Mijajlovic and Biggs constrained the bond lengths and angles during the energy minimization, varying only the dihedral angles (via an evolutionary algorithm). In the study reported here, on the other hand, bond lengths and angles were free to change as all atoms were permitted to move freely when minimizing the structures. The additional localized flexibility means the same three structures are preferred as the surface energy increases but the energies at which they are preferred are shifted relative to when the flexibility is absent.

\subsection{POTENTIAL ENERGY SURFACES FOR 10-ALANINE}

Based on the switching points determined for 10 -alanine, the PES for this molecule were investigated in detail along with the switching transitions for surface energy ratios of $E_{S} / E_{A u}=$ $0.0,0.1$ and 0.2 , which bracket the $\alpha \rightarrow 3_{10}$ transition, and $E_{S} / E_{A u}=4.2,4.4$ and 4.6, which straddle the $3_{10} \rightarrow 2_{7}$ transition. Error! Reference source not found. shows the disconnectivity graphs ${ }^{61}$ for $E_{S} / E_{A u}=0.0,0.1$ and 4.4 ; corresponding graphs for the other surface energy ratios investigated are shown in Figure S3 in Supporting Information. It is immediately obvious from these graphs that the presence of the solid surface greatly increases the complexity of the PES of the system, a phenomenon that has previously been noted as an obstacle to studies of peptidesurface interactions. ${ }^{62}$ It is also clear that the degree of complexity broadly increases with the strength of the peptide-surface interaction. This is reflected in Table 2, which shows the number of minima and saddle points identified through the work reported here. Whilst the number of points identified for each surface energy ratio are considerable, the fact that the solid surface 'roughens' the PES means comprehensive enumeration of all stationary points is unrealistic. ${ }^{62}$ 
However, as outlined in Section 2.2, given the variety of methods used to survey the PES and the convergence of the rate constant of the preferred switching pathway with the number of discovered minima, we are confident that the pathways between the three helix forms of interest here have been comprehensively sampled. 
Absolute potential

energy (kcal/mol)

(a)

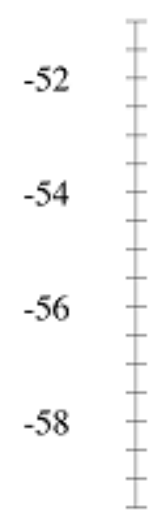

(b)

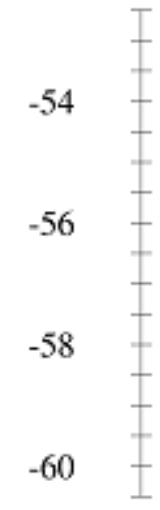

(c)

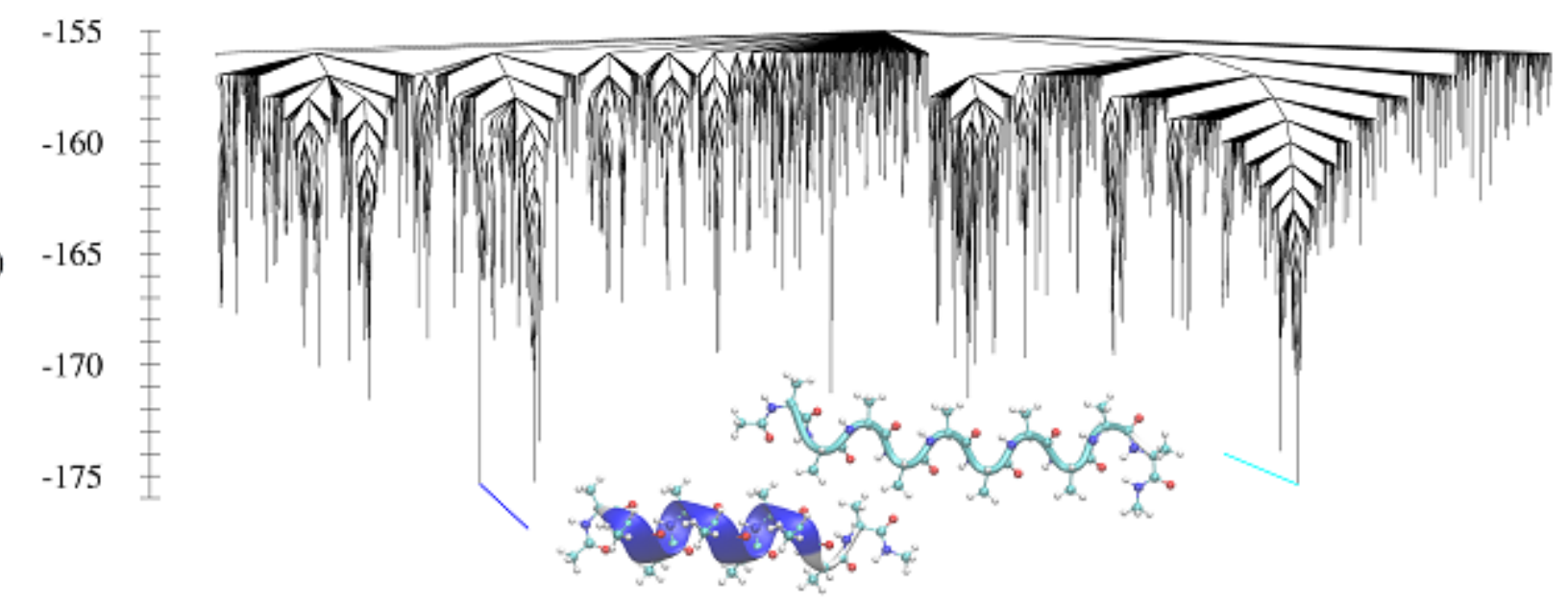


Figure 5. Disconnectivity graphs showing the lowest minima found for 10-alanine at surface energy ratios of: (a) $E_{s} / E_{A u}=0.0$; (b) $E_{s} / E_{A u}=0.1$ and (c) $E_{s} / E_{A u}=4.4$. The minima corresponding to the minimum-energy $\alpha$ - (magenta), $3{ }_{10}$ - (blue) and 27 -helices (cyan) are highlighted.

Table 2. Number of stationary points discovered for the PES of 10-alanine when in the gas phase and adsorbed on surfaces of various surface energies considered in detail here.

\begin{tabular}{|c|c|c|}
\hline \multirow{2}{*}{$\begin{array}{c}\text { surface energy } \\
\text { ratio, } E_{S} / E_{A u}\end{array}$} & \multicolumn{2}{|c|}{ number of } \\
\cline { 2 - 3 } & minima & saddles \\
\hline 0.0 & 6364 & 8288 \\
\hline 0.1 & 6448 & 9064 \\
\hline 0.2 & 7194 & 10116 \\
\hline 4.2 & 28361 & 38331 \\
\hline 4.4 & 24791 & 32825 \\
\hline 4.6 & 25681 & 33357 \\
\hline
\end{tabular}

The termini in the disconnectivity graphs correspond to minima whilst the nodes represent the energy barrier between two minima or groups of minima. ${ }^{61}$ The appearance of a graph can then be used to draw inferences about the underlying PES. ${ }^{63}$ The gas-phase disconnectivity graph shown in Error! Reference source not found.(a) exhibits a classic 'funnel' structure with a number of shallow minima guiding the system down to the global minimum $\alpha$-helix. The 3 10- $^{-}$ helix is one of those 'guiding' minima, separated by a small energy barrier. Error! Reference source not found.(b), which displays the graph at a low surface energy, $E_{S} / E_{A u}=0.1$, where the $\alpha$ - and $3{ }_{10}$-helixes are favored, is similarly shaped to the gas-phase graph but with a number of minima of similar energies, corresponding to these helixes at different orientations to the 
surface. The lower part of this graph resembles the 'banyan tree' structure proposed by Wales and co-workers, ${ }^{63}$ and indicates that at any time the molecule may take any of these structures with similar probabilities.

Error! Reference source not found.(c) shows the disconnectivity graph at a high surface energy, $E_{S} / E_{A u}=4.4$, at which the $310^{-}$and 27 -helixes have similar energies. This exhibits a multi-funnel structure with the most prominent funnel leading down to the ${ }_{7}$-helix. Another funnel contains the $3{ }_{10}$-helix along with another competing minimum, which represents a stable $3{ }_{10} / 27$ hybrid we will return to below. There are several further funnels that indicate other forms 10-alanine may possibly take, albeit with higher potential energy. The relative wideness of the 27 -funnel indicates that the 27 -helix may be more favored when entropy is taken into consideration.

Comparing Error! Reference source not found.(b-c) with the corresponding graphs in Figure S3 indicates the PES do not change dramatically as the switching points are crossed. This suggests that the switching mechanism will be similar in either direction (i.e. from $3_{10} \rightarrow 27$ and from $2_{7} \rightarrow 3_{10}$, for example) and, thus, are not ratchet-like. This absence of a ratchet-like switching may well arise from the symmetry ${ }^{64}$ of polyalanine. This possibility could potentially be revealed by applying the analysis undertaken here to helix-forming asymmetric peptides.

\subsection{SWITCHING TRANSITIONS FOR 10-ALANINE}

The transition path identified for the change between the $\alpha$-helix and $3_{10}$-helix at $E_{S} / E_{A u}=0.1$ is illustrated in Figure 6, with inset figures showing major intermediates and transition states. The switch from the marginally more favored $\alpha$-helix structure to the $3{ }_{10}$-helix was found to occur in three distinct stages. Firstly, the $\alpha$-helix rolls about its longitudinal axis to assume a slightly less stable position on the surface, but one from which the 310 -helix is more readily 
accessible (stage I): the $3^{\text {rd }}$ and $7^{\text {th }}$ residues are nearest to the surface, compared to the starting structure where the $4^{\text {th }}$ and $8^{\text {th }}$ residues are nearest. In the next step, the two turns closest to the $\mathrm{N}$-terminus switch from an $\alpha$ to a $3{ }_{10}$ configuration, forming a marginally stable intermediate between the two helixes (stage II). Finally, the remaining $\alpha$-helical residues, which are adjacent to the C-terminus, switch to yield a full 310 -helix (stage III); it is this stage that presents the highest potential energy barrier in this switching process.

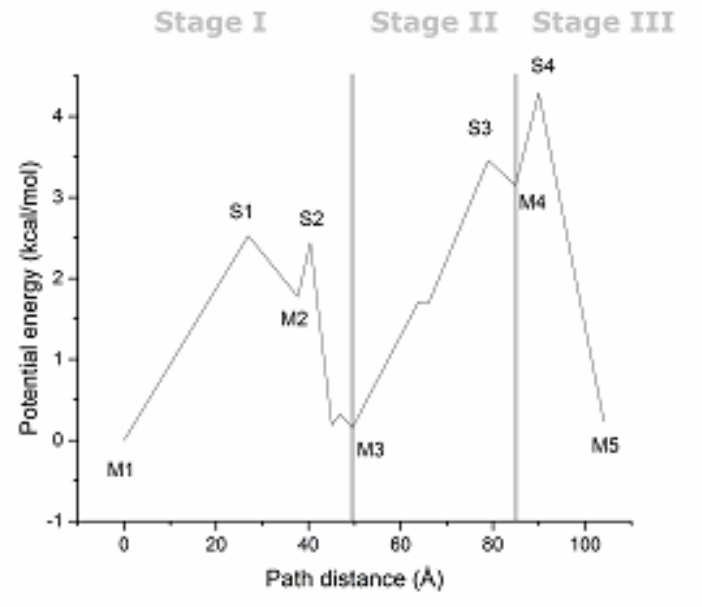

M4

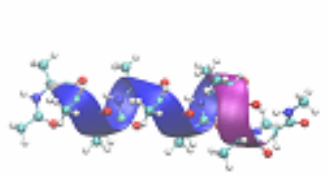

S4

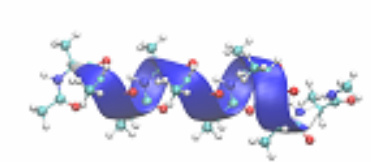

M1

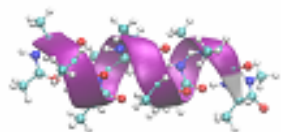

$\mathrm{M} 2$

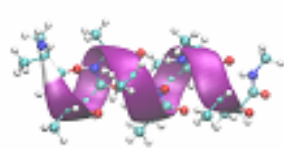

$\mathrm{M} 3$

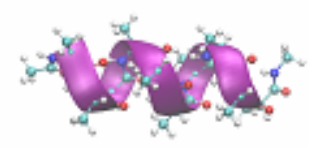

MS

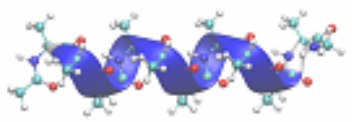

S1

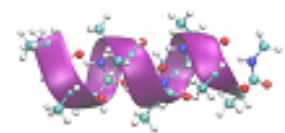

52

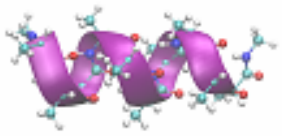

53

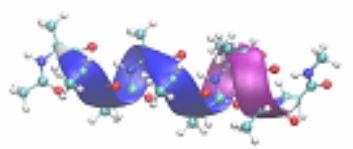

Figure 6. Variation of the potential energy along the transition pathway between the $\alpha$-helix (magenta) and $3{ }_{10}$-helix (blue) at $E_{S} / E_{A u}=0.1$ and images of selected structures along the path, viewed from above the surface. The reference potential energy is that of the minimum-energy $\alpha$ helix adsorbed on the surface. The path distance is the minimized Euclidean distance between neighboring stationary points. Lines between stationary points are provided as a guide to the eye only. 
The paths for the transitions identified at $E_{S} / E_{A u}=0.0$ (gas-phase 10-alanine) and $E_{S} / E_{A u}=$ 0.2, which are shown in Supporting Information (Figures S4 and S5), are similar in nature, although stage I is omitted in the gas-phase transition for obvious reasons. These figures reveal that the path length increases with the surface energy ratio, in line with the increase in the complexity of the PES (see above).

The transition path identified for the change between the $3{ }_{10}$-helix and 27 -helix at $E_{S} / E_{A u}=$ 4.4 is shown in Figure 7, again with inset figures showing intermediate structures. This path is more complex than that for the $\alpha \rightarrow 33_{10}$ switch at lower surface energy, and involves a number of small changes in conformation with, in many cases, high potential energy barriers in between. However, as with the $\alpha \rightarrow 310$ switch, a three-stage mechanism is apparent, punctuated by two notable intermediates between the helixes. Firstly, the $3{ }_{10}$ structure closest to the C-terminus becomes two 27 -helix turns, forming a $3{ }_{10} / 27$ hybrid of comparable stability to the pure helixes (stage I). Secondly, the remainder of the 310 -helix, near the $\mathrm{N}$-terminus, also switches to 27 , but coiling in the middle of the structure produces a moderately stable 'broken' 27 -helix (stage II). Finally, the segment near the N-terminus flips upside down, producing a full 27-helix (stage III). The highest energy barrier occurs at the final stage, although the energy barriers of all three stages are comparable. 


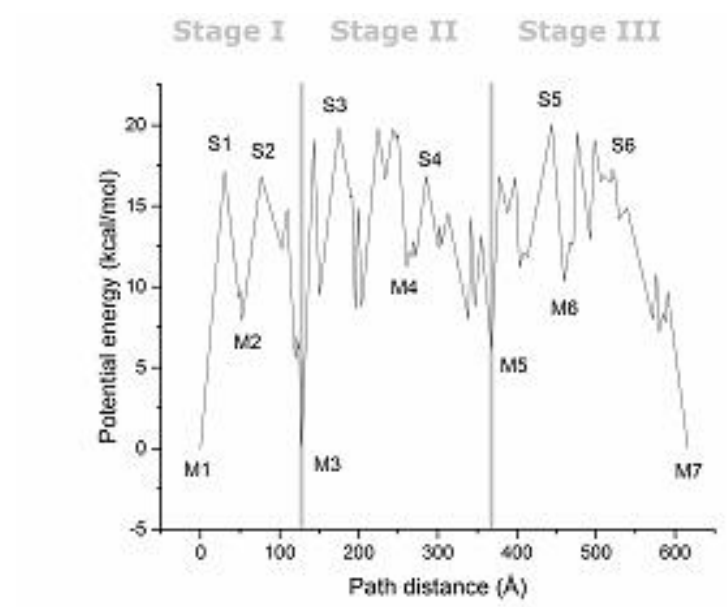

M44

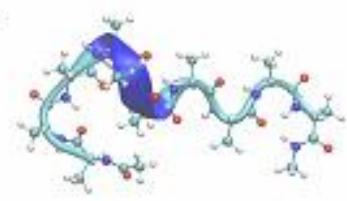

M8

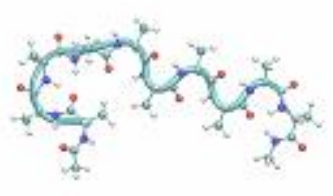

\$4

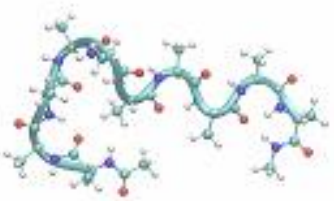

Se

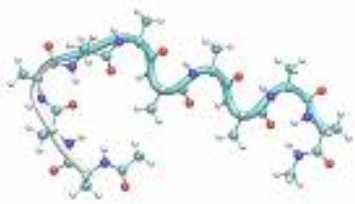

M1

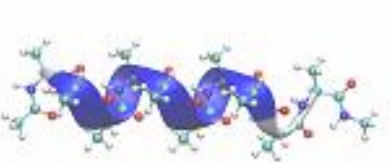

M2

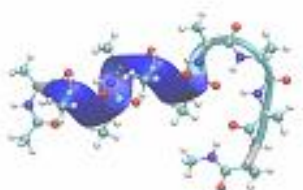

$\mathrm{M} 3$

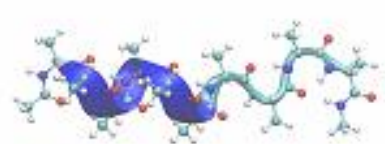

M5

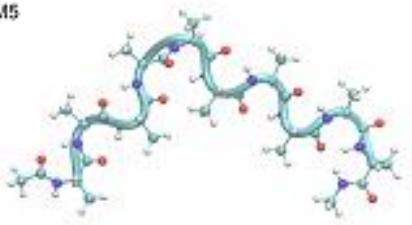

M7

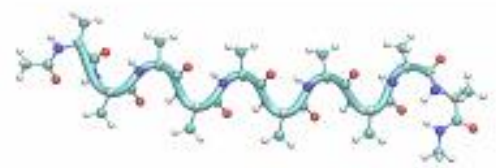

Figure 7. Variation of the potential energy along the transition pathway between the 310 -helix (blue) and 27 -helix (cyan) at $E_{S} / E_{A u}=4.4$ and images of selected structures along the path, viewed from above the surface. The reference potential energy is that of the minimum-energy 310 -helix adsorbed on the surface. The path distance is the minimized Euclidean distance between neighboring stationary points. Lines between stationary points are provided as a guide to the eye only.

Corresponding figures for the transitions identified for $E_{S} / E_{A u}=4.2$ and 4.6 are provided in Supporting Information (Figures S6 and S7). These show the same three-stage process identified here. As with the $\alpha \rightarrow 3_{10}$ switching transition, the path length appears to increase with the surface 
energy ratio. Also, for $E_{S} / E_{A u}=4.6$, the highest energy barrier occurs in stage II, possibly due to the improved stability of 27 -helical structures at the greater surface energy ratio.

It is evident from the results outlined above that the switch from the $3{ }_{10}$-helix to the 27 -helix is significantly more impeded than the $\alpha \rightarrow 310$ switch; the transition path is approximately six times as long and the energy barrier about five times as great. The rate constants, which were evaluated using transition state theory incorporating local harmonic densities of states ${ }^{27}$ quantify in a more direct way the differences in the timescales of each switch. The rate constants for $\alpha \rightarrow 3_{10}$ switching both in the gas phase and at $E_{S} / E_{A u}=0.1$, which are shown in Figure 8 as a function of temperature, are greater than $10^{8} \mathrm{~s}^{-1}$ for temperatures above $200 \mathrm{~K}$. This corresponds to a mean transition time of up to a few nanoseconds, which is well within the capabilities of molecular dynamics simulation. ${ }^{20}$ It is worth noting that an early molecular dynamics study of gas-phase 10-alanine at $300 \mathrm{~K}$ yielded a switch from 310 to $\alpha$ in a few picoseconds, ${ }^{54}$ which corresponds well with our results here. It is also notable that the rates are slower in the presence of the surface by about an order of magnitude, despite the surface favoring the formation of $3_{10}$-helixes; this is possibly another consequence of the increasing complexity of the PES.

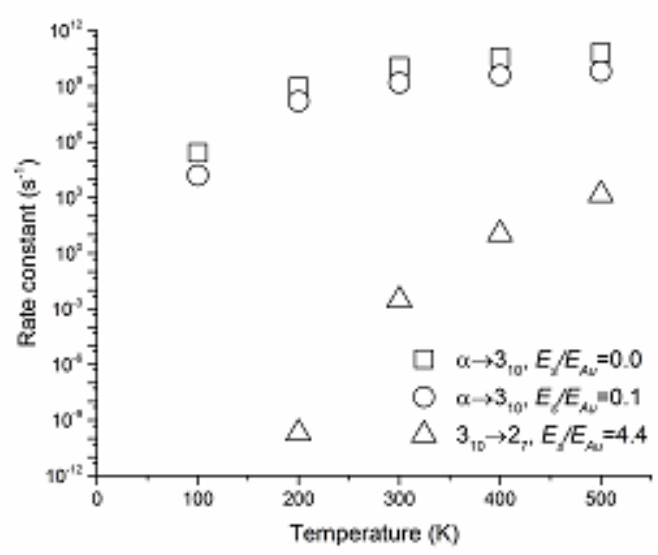


Figure 8. Variation of the rate constants with temperature for the switching transitions at $E_{S} / E_{A u}=0.0,0.1$ and 4.4

The rate constants for $3{ }_{10} \rightarrow 27$ switching at $E_{S} / E_{A u}=4.4$ are far lower than for $\alpha \rightarrow 310$ switching. Even at the maximum temperature sampled of $500 \mathrm{~K}$, the rate constant of approximately $10^{3} \mathrm{~s}^{-1}$ corresponds to a transition time of about a millisecond, which conventional molecular dynamics simulations cannot currently reach. It should be noted that only the rate constant for the most preferred path from the higher-energy minimum to the lower-energy minimum was considered; in reality, the transition is reversible and there are other transition paths and competing structures that would complicate the process and potentially lengthen the necessary simulation timescales.

\subsection{APPROXIMATE ANALYSIS OF COMPUTATIONAL EFFORT}

Because DPS is generally adopted when MD simulation is unable to probe the necessary timescales within a reasonable level of computational resource, ${ }^{27}$ it is worthwhile discussing the effort required to generate the results here. For a system of the size considered here, a full saddle point search incorporating the DNEB and eigenvector following methods took up to a few minutes on a single core of an AMD Opteron 6212 CPU. Thus, to construct a database of the size of those found in this study (c.f. Table 2), at least a few thousand core hours are necessary, and this is further increased by the finding of redundant minima and saddle points, and the extra time taken to read and manage a large database. A conservative estimate of the computational time required is, therefore, around 10,000 core hours. This compares unfavorably with MD for the $\alpha \rightarrow 310$ switch, where MD simulations of the order of 5 core hours on these Opteron CPUs would be sufficient. In the case of the $3{ }_{10} \rightarrow 2_{7}$ switch, on the other hand, the MD simulations time would increase to the order of 10 million core hours even at $500 \mathrm{~K}$, making it entirely 
impractical; DPS is the only viable option. Although the computational time for DPS compares unfavorably for simpler systems such as the ones we considered here at lower surface energies, it is worth noting that this form of analysis is embarrassingly parallelizable (many saddle point searches can be run essentially independently) and it can yield insights that are difficult to gain from MD alone as demonstrated by the present study.

A related point of discussion is how DPS scales with the size of the adsorbed peptide. It is known that the number of stationary points on a potential energy surface generally has an exponential relationship with the size of the system. ${ }^{65}$ This study also demonstrates the effect of the surface in increasing the complexity of the potential energy surface. This suggests that although larger biomolecules have been studied using DPS in the past, ${ }^{33}$ studying the adsorption of such molecules may present greater challenges. Further study will, however, be required before this is fully understood.

\section{CONCLUSIONS}

Three known helical structures formed by polyalanine at a gas/solid interface were subjected to energy minimization and compared. In agreement with past findings, ${ }^{35}$ a switching phenomenon was observed from $\alpha$-helix to 310 -helix to 27 -helix, in order of increasing strength of the surfacepeptide interaction. A discrete path sampling procedure was used to investigate this switching process for 10-alanine, and general stage-by-stage mechanisms for the $\alpha \rightarrow 3_{10}$ and $3_{10} \rightarrow 27$ switches were identified. The former switch is a relatively simple and energetically accessible process that involves the molecule 'rolling' on the surface and then transforming piecewise from an $\alpha$-helix to a $3_{10}$-helix. The $3_{10} \rightarrow 27$ switch is more complex, involving a number of small changes and intermediates, with high energy barriers and low rate constants. As a consequence, while the $\alpha \rightarrow 33_{10}$ switch occurs rapidly enough that it may easily be observed using molecular 
dynamics simulation, the $3_{10} \rightarrow 2_{7}$ switch will normally be beyond current molecular dynamics simulation capabilities. The information gleaned about the $3_{10} \rightarrow 2_{7}$ transition demonstrates the possibilities inherent in this form of study, which could well be applied to other cases of biomolecule adsorption that occur over long periods of time.

This study largely focused on the PES; entropic effects were only included when calculating rate constants, and these only took into consideration the most favored path in one direction. The disconnectivity graphs of the PES obtained here suggest that entropy may have some influence at least quantitatively; we are, therefore, working on including entropic contributions more fully, ${ }^{66-}$ ${ }^{67}$ which we will report on in the future. We will also investigate the variation of complexity and computational effort as a function of the peptide size and other peptide characteristics. Finally, as

it is anticipated that a solvent will affect the switching behavior explored here, ${ }^{35}$ incorporation of solvent in DPS analysis is of interest. Unfortunately, this is a major challenge at present, ${ }^{62}$ and will need to await further methodological developments.

SUPPORTING INFORMATION

S1: Discrete path sampling procedure. S2: Algorithms and schemes used. S3: Development of preferred paths. S4: Additional disconnectivity graphs. S5: Additional transition paths. This material is available free of charge via the Internet at http://pubs.acs.org.

\section{ACKNOWLEDGMENT}

J.A.R. is grateful to the University of Adelaide for the receipt of an Australian Postgraduate Award. The support of the Australian Research Council Discovery Program (DP130101714) is gratefully acknowledged. The authors acknowledge the computational resources provided by 
eResearchSA, including a project-specific allocation of resources on the NeCTAR Research Cloud, and the Phoenix HPC facility at the University of Adelaide.

\section{REFERENCES}

1. Nakanishi, K.; Sakiyama, T.; Imamura, K. On the Adsorption of Proteins on Solid Surfaces, a Common but Very Complicated Phenomenon. J. Biosci. Bioeng. 2001, 91, 233-244.

2. Gray, J. J. The Interaction of Proteins with Solid Surfaces. Curr. Opin. Struct. Biol. 2004, $14,110-115$.

3. Rabe, M.; Verdes, D.; Seeger, S. Understanding Protein Adsorption Phenomena at Solid Surfaces. Adv. Colloid Interface Sci. 2011, 162, 87-106.

4. Nel, A. E.; Madler, L.; Velegol, D.; Xia, T.; Hoek, E. M. V.; Somasundaran, P.; Klaessig, F.; Castranova, V.; Thompson, M. Understanding Biophysicochemical Interactions at the NanoBio Interface. Nat. Mater. 2009, 8, 543-557.

5. Mahmoudi, M.; Lynch, I.; Ejtehadi, M. R.; Monopoli, M. P.; Bombelli, F. B.; Laurent, S. Protein-Nanoparticle Interactions: Opportunities and Challenges. Chem. Rev. 2011, 111, 56105637.

6. Seker, U. O. S.; Demir, H. V. Material Binding Peptides for Nanotechnology. Molecules 2011, 16, 1426-1451.

7. Laera, S.; Ceccone, G.; Rossi, F.; Gilliland, D.; Hussain, R.; Siligardi, G.; Calzolai, L. Measuring Protein Structure and Stability of Protein-Nanoparticle Systems with Synchrotron Radiation Circular Dichroism. Nano Lett. 2011, 11, 4480-4484. 
8. Coppage, R.; Slocik, J. M.; Briggs, B. D.; Frenkel, A. I.; Heinz, H.; Naik, R. R.; Knecht, M. R. Crystallographic Recognition Controls Peptide Binding for Bio-Based Nanomaterials. J. Am. Chem. Soc. 2011, 133, 12346-12349.

9. Cho, N.-H.; Cheong, T.-C.; Min, J. H.; Wu, J. H.; Lee, S. J.; Kim, D.; Yang, J.-S.; Kim, S.; Kim, Y. K.; Seong, S.-Y. A Multifunctional Core-Shell Nanoparticle for Dendritic CellBased Cancer Immunotherapy. Nat. Nanotechnol. 2011, 6, 675-682.

10. Kumar, A.; Ma, H.; Zhang, X.; Huang, K.; Jin, S.; Liu, J.; Wei, T.; Cao, W.; Zou, G.; Liang, X.-J. Gold Nanoparticles Functionalized with Therapeutic and Targeted Peptides for Cancer Treatment. Biomaterials 2012, 33, 1180-1189.

11. Soum, C.; Rubio-Albenque, S.; Fery-Forgues, S.; Déléris, G.; Alouini, M.-A.; Berthelot, T. Supramolecular Peptide/Surface Assembly for Monitoring Proteinase Activity and Cancer Diagnosis. ACS Appl. Mater. Interfaces 2015, 7, 16967-16975.

12. Zhang, M.; Yin, B.-C.; Wang, X.-F.; Ye, B.-C. Interaction of Peptides with Graphene Oxide and Its Application for Real-Time Monitoring of Protease Activity. Chem. Commun. 2011, 47, 2399-2401.

13. Mannoor, M. S.; Tao, H.; Clayton, J. D.; Sengupta, A.; Kaplan, D. L.; Naik, R. R.; Verma, N.; Omenetto, F. G.; McAlpine, M. C. Graphene-Based Wireless Bacteria Detection on Tooth Enamel. Nat. Commun. 2012, 3, 763.

14. Qu, Y.; Ma, M.; Wang, Z.; Zhan, G.; Li, B.; Wang, X.; Fang, H.; Zhang, H.; Li, C. Sensitive Amperometric Biosensor for Phenolic Compounds Based on Graphene-Silk Peptide/Tyrosinase Composite Nanointerface. Biosens. Bioelectron. 2013, 44, 85-88. 
15. Huotari, A.; Xu, W.; Mönkäre, J.; Kovalainen, M.; Herzig, K.-H.; Lehto, V.-P.; Järvinen, K. Effect of Surface Chemistry of Porous Silicon Microparticles on Glucagon-Like Peptide-1 (GLP-1) Loading, Release and Biological Activity. Int. J. Pharm. 2013, 454, 67-73.

16. Meyers, S. R.; Grinstaff, M. W. Biocompatible and Bioactive Surface Modifications for Prolonged in Vivo Efficacy. Chem. Rev. 2011, 112, 1615-1632.

17. Mu, Y.; Tang, B.; Yu, M. Length-Dependent $\beta$-Sheet Growth Mechanisms of Polyalanine Peptides in Water and on Hydrophobic Surfaces. Phys. Rev. E: Stat., Nonlinear, Soft Matter Phys. 2014, 89, 032711.

18. Bellucci, L.; Ardèvol, A.; Parrinello, M.; Lutz, H.; Lu, H.; Weidner, T.; Corni, S. The Interaction with Gold Suppresses Fiber-Like Conformations of the Amyloid $\beta$ (16-22) Peptide. Nanoscale 2016, 8, 8737-8748.

19. Latour, R. A. Molecular Simulation of Protein-Surface Interactions: Benefits, Problems, Solutions, and Future Directions (Review). Biointerphases 2008, 3, FC2-FC12.

20. Ozboyaci, M.; Kokh, D. B.; Corni, S.; Wade, R. C. Modeling and Simulation of ProteinSurface Interactions: Achievements and Challenges. Q. Rev. Biophys. 2016, 49, e4.

21. Biswas, P. K.; Vellore, N. A.; Yancey, J. A.; Kucukkal, T. G.; Collier, G.; Brooks, B. R.; Stuart, S. J.; Latour, R. A. Simulation of Multiphase Systems Utilizing Independent Force Fields to Control Intraphase and Interphase Behavior. J. Comput. Chem. 2012, 33, 1458-1466.

22. Mijajlovic, M.; Penna, M. J.; Biggs, M. J. Free Energy of Adsorption for a Peptide at a Liquid/Solid Interface via Nonequilibrium Molecular Dynamics. Langmuir 2013, 29, 2919-2926. 
23. Bellucci, L.; Corni, S. Interaction with a Gold Surface Reshapes the Free Energy Landscape of Alanine Dipeptide. J. Phys. Chem. C 2014, 118, 11357-11364.

24. Penna, M. J.; Mijajlovic, M.; Biggs, M. J. Molecular-Level Understanding of Protein Adsorption at the Interface Between Water and a Strongly Interacting Uncharged Solid Surface. J. Am. Chem. Soc. 2014, 136, 5323-5331.

25. Penna, M. J.; Mijajlovic, M.; Tamerler, C.; Biggs, M. J. Molecular-Level Understanding of the Adsorption Mechanism of a Graphite-Binding Peptide at the Water/Graphite Interface. Soft Matter 2015, 11, 5192-5203.

26. Ozboyaci, M.; Kokh, D. B.; Wade, R. C. Three Steps to Gold: Mechanism of Protein Adsorption Revealed by Brownian and Molecular Dynamics Simulations. Phys. Chem. Chem. Phys. 2016, 18, 10191-10200.

27. Wales, D. J. Discrete Path Sampling. Mol. Phys. 2002, 100, 3285-3305.

28. Trygubenko, S. A.; Wales, D. J. Kinetic Analysis of Discrete Path Sampling Stationary Point Databases. Mol. Phys. 2006, 104, 1497-1507.

29. Wales, D. J. Calculating Rate Constants and Committor Probabilities for Transition Networks by Graph Transformation. J. Chem. Phys. 2009, 130, 204111.

30. Calvo, F.; Doye, J. P. K.; Wales, D. J. Energy Landscapes of Colloidal Clusters: Thermodynamics and Rearrangement Mechanisms. Nanoscale 2012, 4, 1085-1100.

31. Farrell, J. D.; Lines, C.; Shepherd, J. J.; Chakrabarti, D.; Miller, M. A.; Wales, D. J. Energy Landscapes, Structural Topologies and Rearrangement Mechanisms in Clusters of Dipolar Particles. Soft Matter 2013, 9, 5407-5416. 
32. Neelamraju, S.; Oakley, M. T.; Johnston, R. L. Chiral Effects on Helicity Studied via the Energy Landscape of Short (D, L)-Alanine Peptides. J. Chem. Phys. 2015, 143, 165103.

33. Prentiss, M. C.; Wales, D. J.; Wolynes, P. G. The Energy Landscape, Folding Pathways and the Kinetics of a Knotted Protein. PLoS Comput. Biol. 2010, 6, e1000835.

34. Carr, J. M.; Whittleston, C. S.; Wade, D. C.; Wales, D. J. Energy Landscapes of a Hairpin Peptide Including NMR Chemical Shift Restraints. Phys. Chem. Chem. Phys. 2015, 17, 2025020258.

35. Mijajlovic, M.; Biggs, M. J. Study of Conformational Switching in Polyalanine at Solid Surfaces Using Molecular Simulation. J. Phys. Chem. C 2007, 111, 15839-15847.

36. Humphrey, W.; Dalke, A.; Schulten, K. VMD: Visual Molecular Dynamics. J. Mol. Graphics 1996, 14, 33-38.

37. Cornell, W. D.; Cieplak, P.; Bayly, C. I.; Gould, I. R.; Merz, K. M.; Ferguson, D. M.; Spellmeyer, D. C.; Fox, T.; Caldwell, J. W.; Kollman, P. A. A Second Generation Force Field for the Simulation of Proteins, Nucleic Acids, and Organic Molecules. J. Am. Chem. Soc. 1995, $117,5179-5197$.

38. Steele, W. A. The Physical Interaction of Gases with Crystalline Solids: I. Gas-Solid Energies and Properties of Isolated Adsorbed Atoms. Surf. Sci. 1973, 36, 317-352.

39. Mahaffy, R.; Bhatia, R.; Garrison, B. J. Diffusion of a Butanethiolate Molecule on a Au $\{111\}$ Surface. J. Phys. Chem. B 1997, 101, 771-773.

40. Lide, D. R. CRC Handbook of Chemistry and Physics; 89th ed.; CRC Press: Cleveland, Ohio, 2009. 
41. Liu, D. C.; Nocedal, J. On the Limited Memory BFGS Method for Large Scale Optimization. Math. Prog. 1989, 45, 503-528.

42. Wales, D. J.; Doye, J. P. K. Global Optimization by Basin-Hopping and the Lowest Energy Structures of Lennard-Jones Clusters Containing up to 110 Atoms. J. Phys. Chem. A 1997, 101, 5111-5116.

43. Carr, J. M.; Trygubenko, S. A.; Wales, D. J. Finding Pathways Between Distant Local Minima. J. Chem. Phys. 2005, 122, 234903.

44. Carr, J. M.; Wales, D. J. Global Optimization and Folding Pathways of Selected $\alpha$ Helical Proteins. J. Chem. Phys. 2005, 123, 234901.

45. Strodel, B.; Whittleston, C. S.; Wales, D. J. Thermodynamics and Kinetics of Aggregation for the GNNQQNY Peptide. J. Am. Chem. Soc. 2007, 129, 16005-16014.

46. Wales Group Home Page. http://www-wales.ch.cam.ac.uk/ (accessed February 2, 2017).

47. Trygubenko, S. A.; Wales, D. J. A Doubly Nudged Elastic Band Method for Finding Transition States. J. Chem. Phys. 2004, 120, 2082-2094.

48. Munro, L. J.; Wales, D. J. Defect Migration in Crystalline Silicon. Phys. Rev. B: Condens. Matter Mater. Phys. 1999, 59, 3969-3980.

49. Carr, J. M.; Wales, D. J. In Latest Advances in Atomic Cluster Collisions: Structure and Dynamics from the Nuclear to the Biological Scale; Connerade, J.-P.; Solov'yov, A., Eds.; Imperial College Press: London, 2008; pp 321-330. 
50. Brooks, B. R.; Brooks, C. L.; MacKerell, A. D.; Nilsson, L.; Petrella, R. J.; Roux, B.; Won, Y.; Archontis, G.; Bartels, C.; Boresch, S., et al. CHARMM: The Biomolecular Simulation Program. J. Comput. Chem. 2009, 30, 1545-1614.

51. Schafer, L.; Newton, S. Q.; Cao, M.; Peeters, A.; Van Alsenoy, C.; Wolinski, K.; Momany, F. A. Evaluation of the Dipeptide Approximation in Peptide Modeling by Ab Initio Geometry Optimizations of Oligopeptides. J. Am. Chem. Soc. 1993, 115, 272-280.

52. Topol, I. A.; Burt, S. K.; Deretey, E.; Tang, T.-H.; Perczel, A.; Rashin, A.; Csizmadia, I. G. $\alpha$-and 310-Helix Interconversion: A Quantum-Chemical Study on Polyalanine Systems in the Gas Phase and in Aqueous Solvent. J. Am. Chem. Soc. 2001, 123, 6054-6060.

53. Park, C.; Goddard, W. A. Stabilization of $\alpha$-Helices by Dipole-Dipole Interactions Within a-Helices. J. Phys. Chem. B 2000, 104, 7784-7789.

54. Zhang, L.; Hermans, J. 310 Helix Versus $\alpha$-Helix: A Molecular Dynamics Study of Conformational Preferences of Aib and Alanine. J. Am. Chem. Soc. 1994, 116, 11915-11921.

55. Okamoto, Y.; Hansmann, U. H. E. Thermodynamics of Helix-Coil Transitions Studied by Multicanonical Algorithms. J. Phys. Chem. 1995, 99, 11276-11287.

56. Mortenson, P. N.; Evans, D. A.; Wales, D. J. Energy Landscapes of Model Polyalanines. J. Chem. Phys. 2002, 117, 1363-1376.

57. Rossi, M.; Blum, V.; Kupser, P.; von Helden, G.; Bierau, F.; Pagel, K.; Meijer, G.; Scheffler, M. Secondary Structure of Ac-Ala n-LysH+ Polyalanine Peptides $(n=5,10,15)$ in Vacuo: Helical or Not? J. Phys. Chem. Lett. 2010, 1, 3465-3470. 
58. Mu, Y. Effects of Surface Hydrophobicity on the Conformational Changes of Polypeptides of Different Length. Phys. Rev. E: Stat., Nonlinear, Soft Matter Phys. 2011, 84, 031906.

59. Sek, S.; Tolak, A.; Misicka, A.; Palys, B.; Bilewicz, R. Asymmetry of Electron Transmission Through Monolayers of Helical Polyalanine Adsorbed on Gold Surfaces. J. Phys. Chem. B 2005, 109, 18433-18438.

60. Hu, Q.; Wang, P.; Laskin, J. Effect of the Surface on the Secondary Structure of Soft Landed Peptide Ions. Phys. Chem. Chem. Phys. 2010, 12, 12802-12810.

61. Becker, O. M.; Karplus, M. The Topology of Multidimensional Potential Energy Surfaces: Theory and Application to Peptide Structure and Kinetics. J. Chem. Phys. 1997, 106, 1495-1517.

62. Wright, L. B.; Walsh, T. R. Efficient Conformational Sampling of Peptides Adsorbed onto Inorganic Surfaces: Insights from a Quartz Binding Peptide. Phys. Chem. Chem. Phys. 2013, 15, 4715-4726.

63. Wales, D. J.; Miller, M. A.; Walsh, T. R. Archetypal Energy Landscapes. Nature 1998, 394, 758-760.

64. Cheng, C.; McGonigal, P. R.; Stoddart, J. F.; Astumian, R. D. Design and Synthesis of Nonequilibrium Systems. ACS Nano 2015, 9, 8672-8688.

65. Wales, D. J. Energy Landscapes: Applications to Clusters, Biomolecules and Glasses; Cambridge University Press: Cambridge, U.K., 2003. 
66. Krivov, S. V.; Karplus, M. Free Energy Disconnectivity Graphs: Application to Peptide Models. J. Chem. Phys. 2002, 117, 10894-10903.

67. Evans, D. A.; Wales, D. J. Free Energy Landscapes of Model Peptides and Proteins. $J$. Chem. Phys. 2003, 118, 3891-3897. 
TOC GRAPHIC

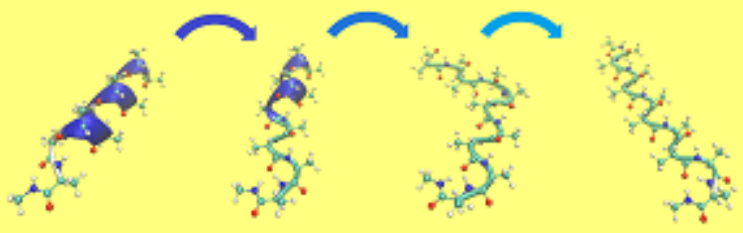

